\title{
Purely chromatic perception of motion in depth: Two eyes as sensitive as one
}

\author{
CHRISTOPHER W. TYLER \\ Smith-Kettlewell Eye Research Institute, San Francisco, California \\ and \\ PATRICK CAVANAGH \\ Harvard University, Cambridge, Massachusetts
}

\begin{abstract}
Motion hyperacuity (phase) thresholds were measured for both lateral and stereoscopic oscillatory motion in both luminance and equiluminant red/green gratings of 2 cycles per degree. Thresholds for lateral chromatic motion did not exhibit the inhibitory fall-off at low temporal frequencies that was found for luminance motion. Phase thresholds for purely chromatic motion were substantially higher than those for luminance gratings, in proportion to the ratio of cone signal modulation, but they could be predicted from the corresponding contrast sensitivities for both types of stimulus. Stereomovement thresholds in luminance gratings showed the stereomovement suppression effect relative to monocular motion sensitivity previously reported for line stimuli, but purely chromatic gratings did not. Together with the lack of an inhibitory fall-off, these results imply that chromatic and luminance motion are processed by different neural pathways, and that the chrominance pathway is capable of supporting a strong percept of stereoscopic motion from purely chromatic gratings.
\end{abstract}

Current evidence suggests that there is a major separation between the processing of color and the processing of motion and depth in primate and human vision; this evidence may be found in both psychophysical (Anstis, 1970; Cavanagh, Tyler, \& Favreau, 1984; Livingstone \& Hubel, 1987; Ramachandran \& Gregory, 1978) and neurophysiological studies (DeYoe \& Van Essen, 1988; Schiller, Charles, \& Logothetis, 1989; Zeki, 1978). We therefore examined the contribution of chrominance pathways to the perception of motion in depth in human vision, since the color/motion separation hypothesis suggests that depth motion should not be visible in purely chromatic stimuli. Moreover, it has been established in a number of studies that visual sensitivity for stereoscopic depth motion has threshold and temporal characteristics that are different from those for monocular, lateral motion (Regan \& Beverley, 1973; Tyler, 1971, 1974, 1975; Tyler \& Foley, 1975). These differences imply separate neural mechanisms for processing lateral and stereoscopic motion perception. This raises the question of whether purely chromatic stimuli can support stereomovement perception.

Cavanagh et al. (1984) showed that lateral motion perception can occur with chromatic, equiluminant gratings, although the motion is perceived as much slower than that of luminance gratings of equal velocity, and this percep-

Please address correspondence to Christopher W. Tyler, SmithKettlewell Eye Research Institute, 2232 Webster Street, San Francisco, CA 94115 . tion may fail altogether at low spatial and temporal frequencies. Can purely chromatic stimuli support the perception of movement in depth, and does that movement have perceptual characteristics similar to those of luminance stereomovement or chromatic lateral movement? How does stereomovement perception compare with the perception of static depth in purely chromatic stimuli, which itself has been shown to occur under some conditions (Comerford, 1974) but not others (Lu \& Fender, 1972)? Finally, does purely chromatic stereomovement have perceptual characteristics similar to those of luminance-based stereomovement? To answer these questions, we measured the thresholds for monocular lateral movement and stereomovement, using red/green gratings carefully matched for equiluminance. It soon became apparent that stereomovement could readily be seen in purely chromatic stimuli, allowing comparison of its characteristics with purely chromatic monocular stimuli, and with the relationships between lateral and depth movement in luminance stimuli.

\section{METHOD}

The stimuli were sinusoidal gratings modulated in color (red/green, see Figure 1A) and/or luminance. They were generated by a $512 \times 512$ pixel resolution graphics system and displayed on a Conrac 5411 monitor. The $x$ and $y$ CIE coordinates of the phosphors were .60 and .35 for red, and .29 and .60 for green. The display subtended $2^{\circ} \times 1^{\circ}$ of visual angle at the $770-\mathrm{cm}$ viewing distance. The stimuli were therefore foveal and not subject to intrusion of luminance signals on the basis of spatial variation of the equiluminance point due to inhomogeneity of the chromatic balance 


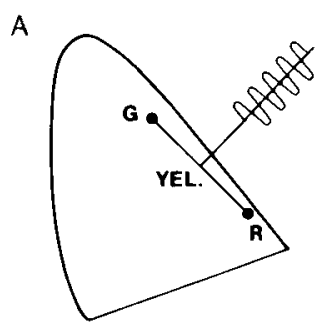

B

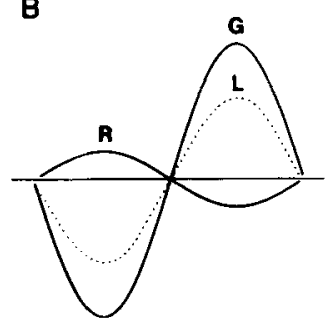

C

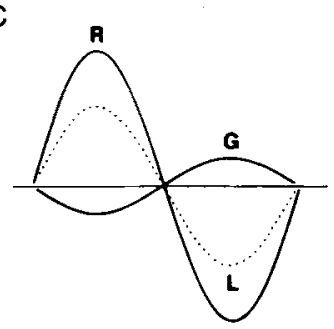

Figure 1A. Depiction of the chromaticity coordinates of the red/green (R/G) stimulus in CIE color space. The dots represent the extremes of possible chromaticity between which the chromatic modulation occurred, as illustrated by the sinusoidal modulation profile. (B and C) Components of the modulation profile where the chromatic contributions are unbalanced so as to produce additional luminance modulation (see text).

from the fovea to the periphery (Livingstone \& Hubel, 1987). Two displays were presented, one above the other on the screen. For dichoptic presentation, the two vertically separated subfields were optically aligned by means of a Risley prism before one eye. The unwanted monocular images that remained above and below the aligned dichoptic images were then obscured by a black cardboard aperture of $2^{\circ} \times 1^{\circ}$ at a distance of $400 \mathrm{~cm}$.

The chromatic gratings were generated by superimposing red and green sinusoidal gratings that were $180^{\circ}$ out of phase. Chrominance modulation was defined as the percentage of the maximum change possible between the red and green phosphors (Figure 1A). The chrominance modulation that we have defined as $100 \%$ is about $46 \%$ of the maximum chromaticity modulation that can be theoretically obtained on the CIE color triangle. The red/green gratings used in these experiments had a chrominance modulation of $70 \%$ of our range, corresponding to $32 \%$ of average modulation of the maximum CIE chromaticity range.

When the red and green modulations were $180^{\circ}$ out of phase, the luminance modulations could be expressed as the difference between their two amplitudes, $\mathbf{R}_{\text {mod }}$ and $\mathbf{G}_{\text {mod }}$ (or the sum of their vectors), divided by the mean luminance of the two waveforms, $R$ and $G$ :

$$
\text { luminance modulation }=\left(R_{\bmod }-G_{\text {mod }}\right) /(R+G) \text {. }
$$

When the stimulus had only luminance modulation, it was a dark/light yellow grating generated by superimposing the red and green waveforms in phase.

Red and green luminances were equated for the 2 cycles per degree (cpd) stimulus in the $2^{\circ} \times 1^{\circ}$ field, using minimum motion heterochromatic photometry (Anstis \& Cavanagh, 1983), with the same spatial and temporal frequencies to be used in the experimental condition. The equiluminance settings were made monocularly for the monocular conditions and binocularly for the binocular conditions. Since both gratings were calibrated to be true sinusoidal modulations of luminance on the monitor screen, their sum at any phase of the cycle produces a constant luminance value if both red and green sine waves have the same luminance modulation amplitude. Positive values of luminance modulation were arbitrarily chosen to represent the case where red modulation amplitude was greater than green, and negative values the reverse. A red/green grating of $70 \%$ chrominance modulation and $10 \%$ luminance modulation, for example, would vary from a yellow-green to a more luminous yellow-orange, while $70 \%$ chrominance and $-10 \%$ luminance modulation would vary from yellow-orange to a slightly more luminous yellow-green (Figures 1B and 1C). A 0\% chrominance, 10\% luminance red/green grating would vary from light to dark yellow. All the displays had a mean luminance of $26 \mathrm{~cd} / \mathrm{m}^{2}$. The gratings were surrounded by $a .08^{\circ}$ border with the same mean luminance as that of the gratings, and they were presented within a fixed aperture on a black background. White fixation spots, $.04^{\circ}$ square at $50 \mathrm{~cd} / \mathrm{m}^{2}$, were presented centrally in the upper and lower gratings.

The left- and right-eye gratings oscillated in opposite directions at a rate set by the experimenter, with an amplitude that could be adjusted by the observer. The gratings moved and reversed direction sinusoidally at the preset rate. The observer's task was to set the oscillation amplitude to threshold by means of a circular knob, while maintaining fixation on the central spot (monocular condition) or fused spots (binocular condition). Before each setting, the experimenter haphazardly rotated the knob so that it was in an ar-

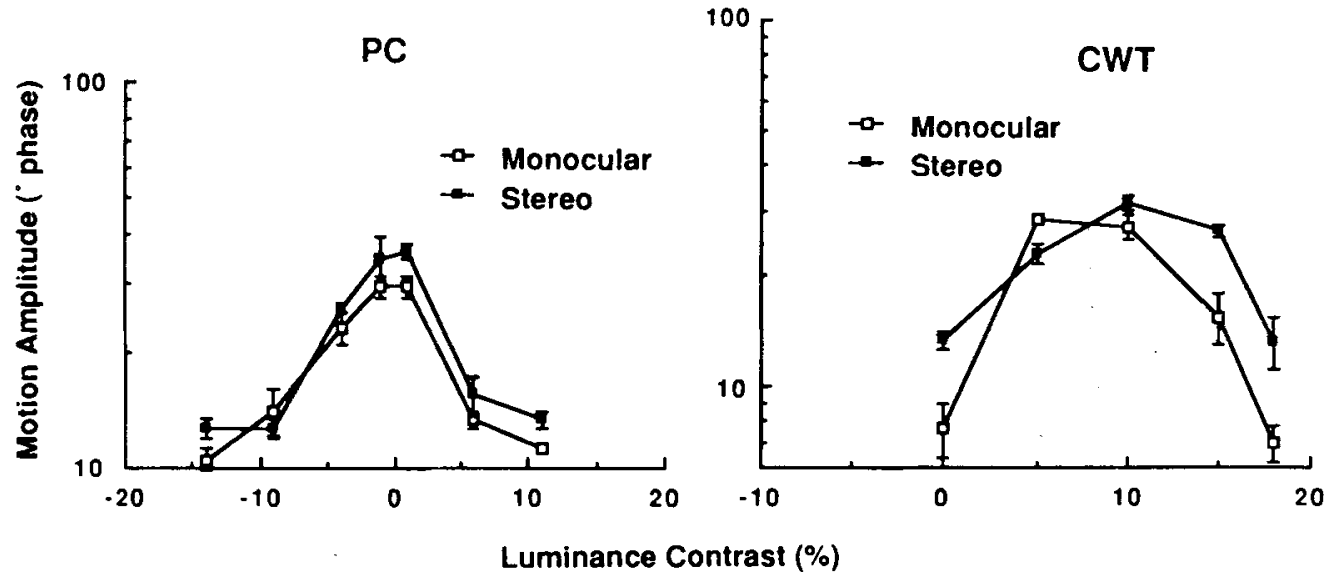

Figure 2. Determination of the equiluminance points for the present stimuli for each observer. Small amounts of spatial luminance modulation (as in Figures $1 \mathrm{~A}-\mathrm{C}$ ) were added around the equiluminance point determined by heterochromatic flicker photometry. The peak values from these data were used for the remaining experiments. 
bitrary position with respect to the threshold position prior to the adjustment. Thus the observer had no information on any trial as to whether the current setting was high or low in relation to any previous settings. The two authors as observers made at least four settings for each condition. Their vision was normal and no color deficits were detected.

Monocular lateral and stereomotion thresholds were measured at a range of luminance contrasts around the predetermined equiluminance point, while the 2-cpd gratings were oscillated at $2 \mathrm{~Hz}$. The results in Figure 2 show that the thresholds rose about a factor of 3 ( $0.5 \mathrm{log}$ units) at equiluminance. The monocular and binocular settings were essentially identical for the chromatic gratings. Motion in depth was still visible for the equiluminous stimuli, although the thresholds $\left(25^{\circ}-35^{\circ}\right.$ of phase, $0.03^{\circ}-0.05^{\circ}$ of visual angle) were quite high.

\section{RESULTS}

\section{Stereomovement Suppression with \\ Luminance Gratings}

It has previously been found with line stimuli that the threshold for stereomovement, or any type of movement percept from disparity modulation, is higher than that for the monocular components of the stimuli (Tyler, 1971, 1974, 1975; Tyler \& Foley, 1975). This "stereomovement suppression" or relative elevation of stereomovement threshold does not necessarily occur for other types of base stimulus, such as random-dot arrays (Regan \& Beverley, 1973). We therefore measured stereoscopic and monocular thresholds for luminance gratings to see whether a similar elevation of stereomovement threshold occurs for grating stimuli. The stereoscopic thresholds were specified in terms of their monocular amplitudes (Figure 3), so that a ratio of greater than 1 means that movement information available when either eye is used alone is lost in stereoscopic view. No additivity of the response from the two eyes is assumed.

The data show that stereomovement threshold elevation of the order of a factor of 2 occurred at all frequencies (Figure 3A), but that it varied across frequency and between observers in a nonsystematic way. Note that if the stereoscopic system used the sum of the information from both eyes, its thresholds would be half those of the monocular conditions (in the method of plotting used for
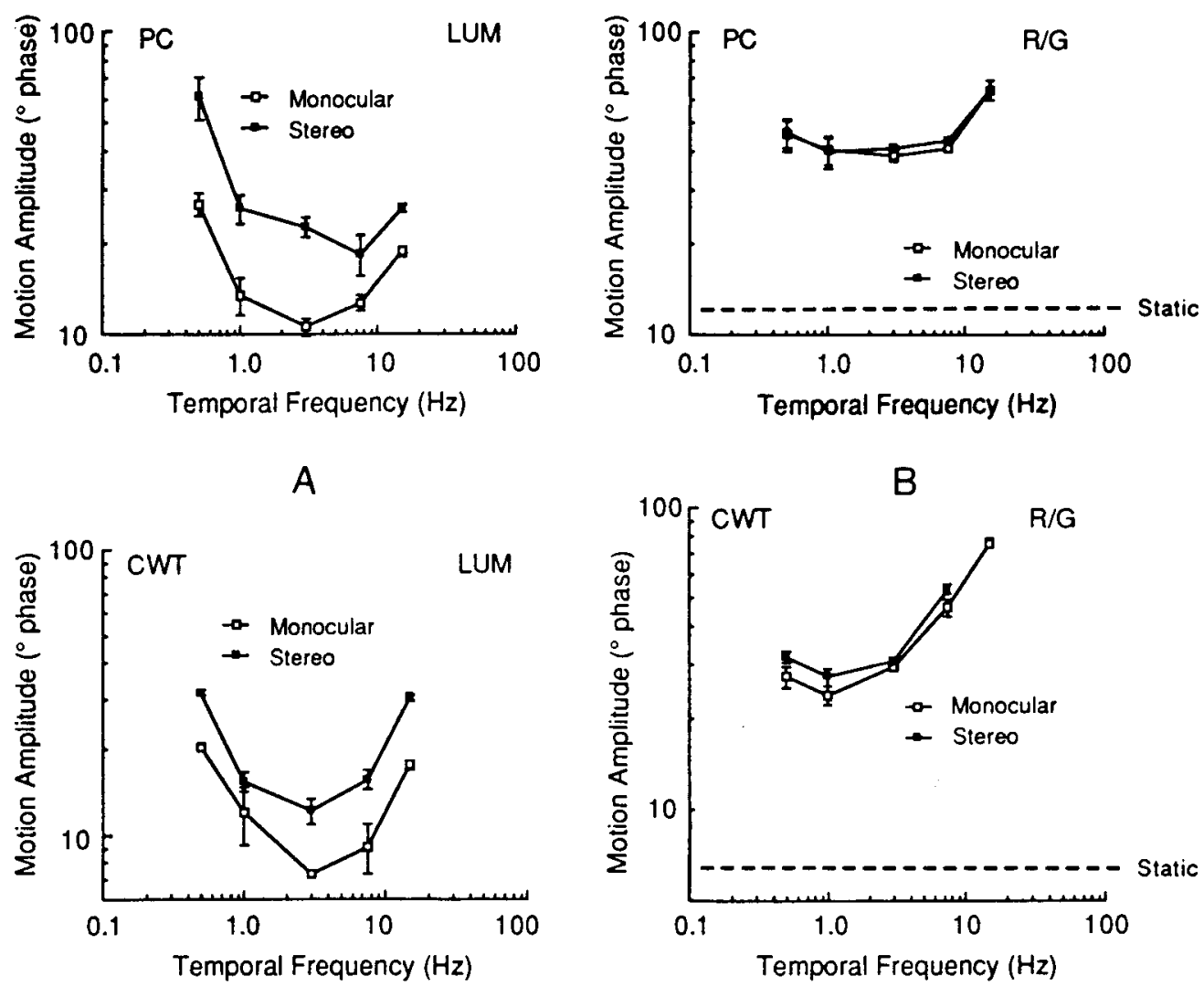

Figure 3. (A) Monocular lateral (open symbols) and stereoscopic depth (filled symbols) oscillation thresholds for a 2-cpd luminance grating as a function of temporal oscillation frequency, for 2 observers. Both types of threshold are plotted in terms of the monocular amplitude of oscillation (i.e., no binocular summation is presumed) in terms of phase angle of the 2-cpd spatial modulation. (B) Monocular lateral (open symbols) and stereoscopic depth oscillation thresholds (filled symbols) for purely chromatic red/green (R/G) gratings of 2-cpd. Note similarity of thresholds under the two conditions. Dashed lines show static stereothresholds relative to the white fixation square. 
Figure 3). Thus, however they are viewed, the stereomotion thresholds for luminance gratings are elevated relative to their monocular components. In this sense, as in the original study (Tyler, 1971), two eyes are less sensitive than one for motion in depth.

\section{Purely Chromatic Stimuli}

The main objective in this study was to measure the perception of stereomovement in purely chromatic stimuli. The procedures for minimizing luminance differences across the sinusoidal chromatic gratings are described in the Method section. In the process, we established that it was possible to perceive stereomovement for purely chromatic gratings at $2 \mathrm{cpd}$ and $2 \mathrm{~Hz}$, and that the thresholds for monocular and stereoscopic movement were very similar near the equiluminance point. We then extended the conditions across the range of temporal frequencies. It was not possible to obtain data at higher spatial frequencies, because the equiluminance point became unstable. This was attributed to small fluctuations in accommodation between the points of focus of the two dominant wavelengths, which differ because of inherent chromatic aberrations. The monocular movement thresholds and stereomovement disparity thresholds were measured for 2-cpd chromatic gratings as a function of temporal frequency.

\section{Monocular Chromatic Motion}

The monocular sinusoidal motion characteristics for purely chromatic gratings (Figure 3B, open symbols) are quite different from those for luminance gratings of the same spatial frequency (Figure 3A, open symbols). At low temporal frequencies, the thresholds were similar (within $0.1 \log$ units at $0.5 \mathrm{~Hz}$ ), but they rapidly deviated as frequency increased. The chromatic motion thresholds improved slightly but then became elevated at higher temporal frequencies, in contrast with the luminance motion thresholds, which improved by about half a log unit before returning toward their original levels by $15 \mathrm{~Hz}$.

This difference in form of the threshold function makes it difficult to attribute the chromatic motion perception to residual stimulation of the luminance pathway. If that were the case, the chromatic motion thresholds should have had the same temporal frequency tuning as the luminance motion thresholds, although elevated in proportion to the luminance leakage ratio. Instead, the temporal functions are rather different in shape, implying that chromatic motion is not processed by the same mechanisms as those for luminance motion.

\section{Chromatic Stereomovement}

For chromatic pairs, the stereomovement thresholds were very similar to the monocular movement thresholds. Thus, adding the counterphase information in the second eye neither raised thresholds, as was the case for many luminance conditions, nor lowered them, as can occur for drifting gratings (Arditi, Anderson, \& Movshon, 1981). This result is a clear difference in the degree of stereo- movement threshold elevation between purely chromatic and purely luminance stimuli.

Note that the similarity in form between the monocular and stereoscopic tuning functions for chromatic stimuli reinforces the difference between temporal properties for chrominance and luminance processing. Now the chromatic stereomovement thresholds actually cross over to become lower at low temporal frequencies than their luminance counterparts. Obviously, residual luminance cues could not account for this result, even if the equiluminance balance was shifted entirely onto one cone pathway. Yet the high-frequency thresholds for chromatic stereomovement were still elevated by about a factor of 3 relative to their luminance counterparts. Thus, for chromatic stereomotion, as for lateral chromatic motion, the temporal properties imply a separate processing mechanism from that for luminance stereomotion.

\section{Static/Dynamic Comparison}

Static depth thresholds were obtained by setting the disparity of the red/green grating so that it would appear alternately just behind and just in front of the white fixation point. Although this task was straightforward, a parallel attempt to obtain monocular position thresholds by setting a peak of the purely chromatic grating position in relation to the fixation point foundered in instability. Under monocular observation, the relative positions of the grating and the fixation point fluctuated unpredictably over a range of at least $180^{\circ}$ of the grating cycle, and attempted threshold settings were unreproducible. This observation also serves to indicate that the static red/green grating remained at the equiluminance point, since the presence of residual luminance cues should have stabilized the percept (as was the case for low-contrast luminance gratings).

Despite the problems with monocular observation, however, static depth thresholds with the purely chromatic gratings viewed stereoscopically were reliable to within an average of $\pm 2^{\circ}$ of phase disparity ( \pm 10 arc sec at $2 \mathrm{cpd}$ ).

The static depth thresholds obtained were about 1 arc $\mathrm{min}$ for red/green at this 2 -cpd condition $\left(10^{\circ}\right.$ of phase). This is quite a respectable performance, considering the low spatial frequency and complete monocular instability of the chromatic gratings. The stereomovement thresholds did not reach as low as the static values at any temporal frequency. The discrepancy was at minimum a factor of 4 for both observers, and increased for higher temporal frequencies.

\section{DISCUSSION}

\section{Lateral Oscillation of Luminance Gratings}

The threshold functions for monocular observation of the luminance grating oscillation have a $U$-shaped form, with peak sensitivity in the region of $5 \mathrm{~Hz}$. This can be related to the sensitivity (reciprocal of threshold) for counterphase modulation of a luminance grating, which has a similar form (Robson, 1966), by a phase vector anal- 

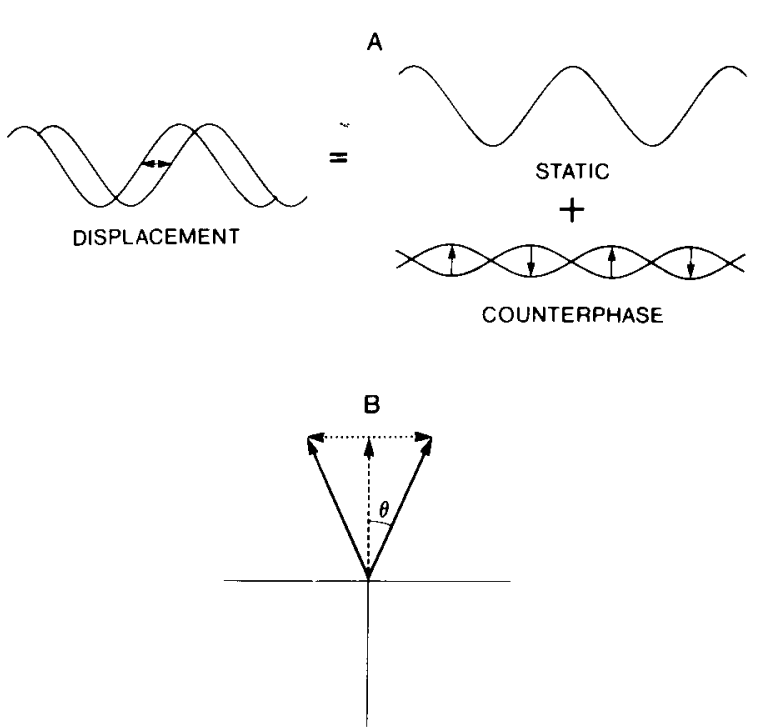

Figure 4. (A) Component diagram of the decomposition of an oscillating grating into a static component and a counterphase modulating component at $90^{\circ}$ of spatial phase. (B) Phase diagram of grating oscillation between two positions, where the vectors each represent a sinusoidal grating of a modulation amplitude defined by the length of the vector and a spatial phase relative to some arbitrary reference (here given as vertical) shown by the vector orientation. Alternation between two positions (solid vectors) may be equivalently described as a static grating (dashed vector) summed with a counterphase modulating grating (dotted vector), showing geometrically how it is always in quadrature $\left(90^{\circ}\right)$ with the static component.

ysis of the grating oscillation. In the case of oscillation of a sinusoidal grating (Figure 4B), the resulting modulation component is itself a sinusoidal modulation of the same spatial frequency formed by the modulation vector (m) about the mean vector corresponding to the static, unmodulated component $(s)$. The length of the modulation vector is half the total modulation contrast and is always at right angles $\left(90^{\circ}\right.$ phase) to the static component. Thus, the detection of grating oscillation may be regarded as a contrast masking paradigm in which the quadraturemodulated component corresponds to the contrast increment against the background of the static component. Data for this kind of task (Lawton \& Tyler, 1986a; Stromeyer \& Klein, 1974) suggest that for a background grating of $10 \%$ contrast, the threshold is elevated by a small factor of about 2 , relative to detection threshold. This value may therefore be used as a prediction for phase threshold in the grating oscillation task.

In the present situation, detection threshold for static gratings occurred at a Michelson contrast of $1 / 400$. For the static grating contrast $(c)$ of $10 \%$, therefore, the previous data suggest that a modulation vector of about twice this value (i.e., 1/200) should be required to account for the masking effect of $10 \%$ static contrast.

From Figure 4, it may be seen that the half-angle of phase $(\phi / 2)$ oscillation is related to the modulation vector by the expression

$$
\sin \phi / 2=\mathbf{m} / c .
$$

For small angles, the sine of an angle is equal to the angle in radians, and

$$
\phi=2 \mathrm{~m} / c \text { radians. }
$$

Insertion of the above estimates for $\mathbf{m}$ and $c$ gives a predicted phase threshold of about $6^{\circ}$, or about 0.5 arc min at $2 \mathrm{cpd}$. The data of Figure $3 \mathrm{~A}$ are in good agreement with this prediction, having minimum monocular phase thresholds of about $7^{\circ}-11^{\circ}$. This implies that motion detection is limited by the same constraints as contrast discrimination. The same conclusion was reached in a study of contrast discrimination using in-phase and quadrature increments of contrast (Lawton \& Tyler, $1986 \mathrm{~b})$. Thus, the availability of motion information in addition to the contrast increment neither confers an advantage at detection threshold nor requires extra contrast for its detection.

\section{Attribution of Low Temporal Sensitivity to Lateral Inhibition}

A proportionate reduction in sensitivity at low temporal frequencies is often attributed to the operation of an inhibitory mechanism with a slower response time than the excitatory response (see, e.g., Kelly, 1971). It is easy to show that this inhibition is a spatial property of the system, as opposed to a purely temporal property independent of spatial configuration. This conclusion follows from the results in Robson (1966) and in subsequent studies that the sensitivity reduction at low temporal frequency is not obtained with modulation of high spatial frequency gratings, but occurs only at spatial frequencies below $3 \mathrm{cpd}$ for foveal viewing. This must mean that the inhibition is mediated by lateral connections over distances greater than 10 arc $\min$ (the reciprocal of the half wavelength at $3 \mathrm{cpd}$ ), which only have the opportunity to attenuate signals from neighboring regions in stimuli with patches larger than 10 arc min. If the stimulus changes to the opposite sign within this distance, as occurs in higher frequency gratings, the inhibition will tend to enhance rather than diminish the response. Another way to put this is to say that the inhibitory network cannot resolve spatial modulation beyond $3 \mathrm{cpd}$ and is therefore inoperative at higher spatial frequencies.

The conclusion from this analysis is that all measurable inhibition is lateral inhibition, since the low-frequency attenuation is eliminated at high spatial frequencies, where only direct temporal inhibition could operate. Since the analysis of Equation 2 suggests that the form of the oscillation thresholds can be attributed to the form of the static contrast thresholds, the same conclusion presumably applies to the oscillation threshold mechanism. A simple model of this inhibitory effect for the motion signal can be developed from a two-pole low-pass filter with onepole subtractive inhibition (Tyler, 1978). The temporal frequency response, $F(i \omega)$, of such a filter combination may be described by 


$$
F(i \omega)=a\left(\frac{1}{1+i t_{1} \omega}\right)^{2}\left(1-\frac{b}{1+i t_{2} \omega}\right),
$$

where $i$ is the square root of $-1, \omega$ is the angular frequency of the oscillation, $a$ is the sensitivity constant, $t_{1}$ and $t_{2}$ are the excitatory and inhibitory time constants, and $b$ is the strength of the inhibition. This function, which is shown as the upper full line in Figure 5, provides a good description of the luminance oscillation threshold data in Figure 3A, as well as the random-dot oscillation thresholds for which it was originally designed (Tyler, 1978).

\section{Lateral Oscillation of Chromatic Gratings}

Chromatic gratings are produced by, and may be regarded as, the sum of two luminance gratings of different dominant wavelengths added in spatial counterphase (Figure 1). The average modulation of the red and green cone classes by these two implicit luminance gratings was $32 \%$ (see the Method section). In the absence of masking effects, this level of chromatic modulation might be expected to produce a sensitivity increase (for small oscillations) in proportion to the increase in effective contrast, as compared with the $10 \%$ luminance gratings. However, the residual static component of the oscillating grating is also increased in proportion to the contrast, as long as the oscillations remain small. To the extent that contrast masking of the oscillating component by the static component follows Weber's law (which is true for such oscillatory motion in the fovea; Norcia, Wesemann, \&

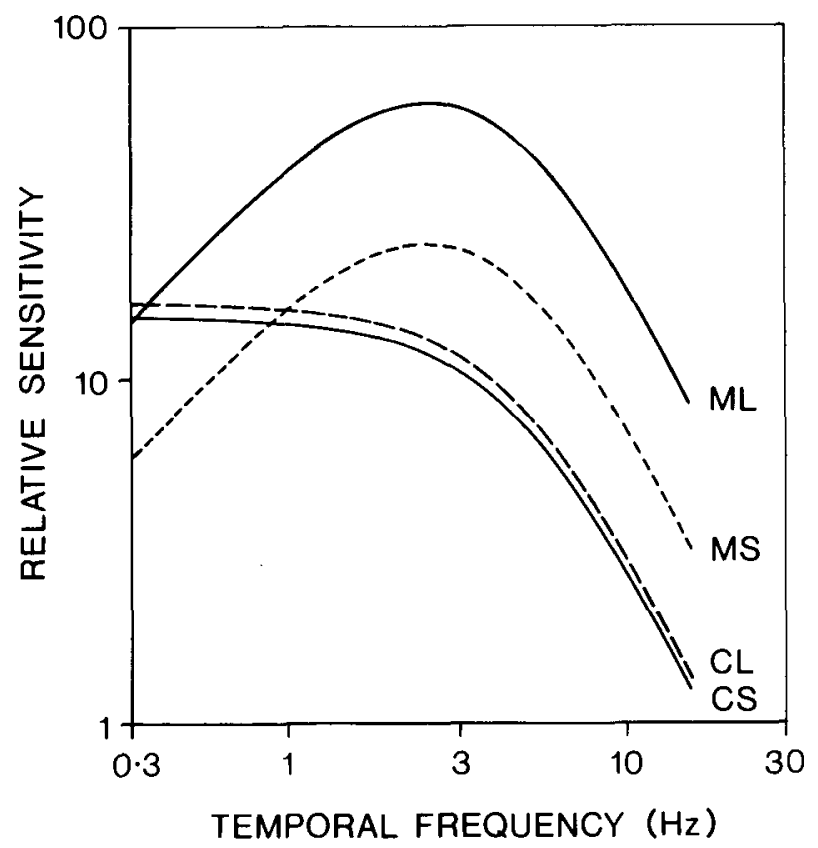

Figure 5. Dynamic model for the processing differences between luminance and chrominance gratings. Temporal frequency response properties are shown for monocular luminance (ML), stereoscopic luminance (SL), monocular chrominance (MC), and stereoscopic chrominance (SC) movement sensitivities (see text for description).
Manny, 1988), the two effects of increased contrast will cancel. Phase thresholds should therefore be roughly independent of contrast, and should remain at about $6^{\circ}$ for the chromatic as well as the luminance modulation. The best measured chromatic thresholds do not reach this value, but were in the range of $25^{\circ}-40^{\circ}$ (Figure 3B). This suggests that the processing for chrominance information is less efficient than for luminance information. This efficiency reduction for the chrominance pathway appears on this simple logic to be about a factor of 6 , which is approximately the threshold elevation expected from the inference that they are mediated by the parvocellular rather than the magnocellular pathway (Kaplan \& Shapley, 1986; Sclar, Maunsell, \& Lennie, 1990).

It is probably best to interpret these results in terms of the chrominance pathway's having both a higher threshold and a reduced level of lateral inhibition. In order to obtain an uncontaminated measure of just the excitatory components of the responses, we can look at the high temporal frequency conditions. In this frequency range, specifically at $7.5 \mathrm{~Hz}$, lateral motion thresholds for the chromatic stimuli are elevated by $0.5-0.7 \mathrm{log}$ units compared to those for luminance stimuli, for the 2 observers. Note, however, that the luminance stimuli were set at only $10 \%$ contrast, approximately 20 times threshold contrast of about $0.5 \%$ at $7.5 \mathrm{~Hz}$ (Robson, 1966), while the chromatic stimuli (at $70 \%$ of the maximum chromatic contrast of the display) were also about 7 times threshold contrast of $10 \%$ at 2 cpd and $8 \mathrm{~Hz}$ (Kelly, 1975; 1979). Thus, the chrominance oscillation thresholds were obtained at $0.5 \mathrm{log}$ units lower effective contrast than the luminance thresholds. We therefore interpret the high frequency threshold elevation of about $0.5 \log$ units of phase as implying that the chrominance and luminance systems have approximately the same inherent sensitivity to motion information, with the measured threshold difference being explained by the underlying difference in sensitivity for the excitatory response to chromatic compared with luminance modulation.

At low temporal frequencies, the chromatic motion thresholds remain approximately constant, in contrast with the inhibitory luminance threshold elevation by about 0.5 $\log$ units at the lowest frequencies compared to their best threshold. The preceding analysis (Equation 3) suggests the following features for chromatic motion and stereomotion processing, which are encapsulated in the model responses of Figure 5 (note that the thresholds of Figure 3 are the reciprocal of the response amplitudes plotted here):

1. The time constant $t_{1}$ is the same for luminance and chromatic motion.

2. The strength of excitation, $a$, is three times greater for monocular luminance than for chromatic motion.

3. The strength of inhibition, $b$, is close to zero for chromatic motion; that is, there is very little rise at low $f$, as contrasted with the full inhibition $(b=1)$ in the luminance case.

4. The strength of excitation, $a$, is about half as much for stereomovement as for monocular oscillation of luminance gratings, but it was equal for the chromatic case. 
A slight reduction in the chromatic sensitivity at low temporal frequencies might be expected from limitations in the attention span required over longer durations for the low temporal frequency conditions. A similar gradual reduction with a slope of $1 / 4$ between 0.05 and $1 \mathrm{~Hz}$ was found for chromatic modulation by Regan and Tyler (1971a). The lack of a full low-frequency fall-off in the chromatic motion sensitivity would imply an absence or relative reduction in the lateral inhibitory component. It further implies that the chromatic response arises at a point in the visual pathway before the operation of this inhibition. The implications of such results for the neural circuitry of color processing are developed in a subsequent section (see Figure 6).

\section{Static Chromatic Thresholds for \\ Position and Depth}

An interesting and unexpected result emerged when we attempted to measure static thresholds for chromatic gratings. It was straightforward to set depth threshold for the $\mathrm{red} /$ green stimulus, but the corresponding monocular task-to set the lateral position to match a particular peak with the white dot-proved to be essentially unquantifiable. What could account for this paradoxical failure in the monocular task when the stereoscopic task could be performed readily?

A possible explanation can be developed from the fact that purely chromatic gratings under image stabilization generate much stronger afterimages, or "sensitivity masks," than do luminance gratings (Kelly, 1979). This result can be applied to free eye movement conditions; the afterimage generated from one fixation would then be summed with the image present in the succeeding fixation position. In sinusoidal stimuli, addition of a stimulus at random to its afterimage produces a summed sinusoidal image of unpredictable phase. The effect of the afterimage is thus to produce apparent random shifts in the position of grating. This effect is particularly marked if the strength of afterimage sinusoid equals that of the stimulus. Since this process would have little effect on the position of a white fixation point, the result would be random shifts in the apparent position of purely chromatic gratings, making the monocular positional thresholds difficult to measure.

However, under binocular observation conditions, the lateral position shifts would tend to be equal in the two eyes, since yoked binocular saccades occur independently of disjunctive vergence movements. Thus the relative disparity between the white fixation point and chromatic grating summed with its afterimage should remain stable, even while the lateral position of the grating is shifting unpredictably. In this way the presence of strong afterimages generates opposite predictions for static monocular and stereoscopic thresholds, which are in accord with the stereoscopic data of Figure 3.

Note that the static stereoscopic thresholds for the purely chromatic stimuli are much lower $\left(10^{\circ}\right.$ of phase) than those for monocular lateral chromatic motion $\left(30^{\circ}-40^{\circ}\right.$ of phase). This can be partly attributed to the presence of equal and opposite shifts in the two eyes for static stereopsis, and partly to the presence of the stable reference point against which the depth is to be judged. A complete explanation for this difference, however, is beyond the scope of the present study.

\section{Chromatic Stereomotion}

Within the accuracy of the data, the detectability of chromatic stereomotion is identical to that for its monocular components at all temporal frequencies. The absence of stereomovement threshold elevation for the chromatic

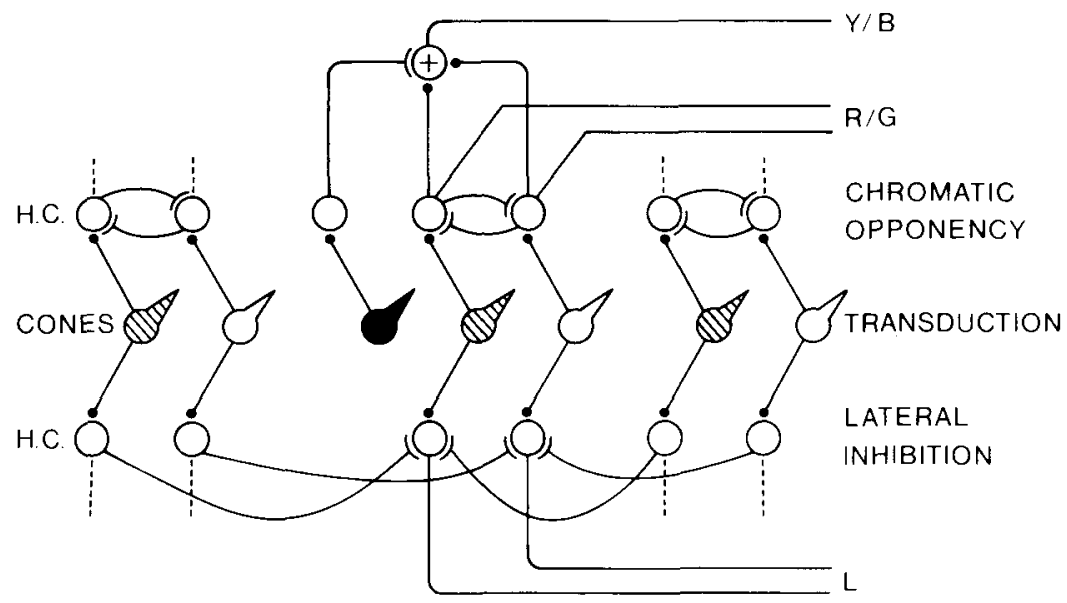

Figure 6. Anatomical model of possible anatomical connections at cone pedicles, which would account for the psychophysical data presented. Cones are shown as sensitive to red (open), green (shaded), and blue (filled) light. Inhibitory connections are indicated in the horizontal cell (H.C.) layers, although the open circles depict bipolar cells. The upper (chrominance) and lower (luminance) outputs from the cone layer are separated for diagrammatic convenience, but not intended to be anatomically segregated. $Y / B=$ yellow/blue; $\mathbf{R} / \mathbf{G}=\mathrm{red} / \mathrm{green}$ outputs. 
stimuli further demonstrates that the perception of stereomotion for these stimuli could not have been mediated by luminance artifacts. At $2 \mathrm{cpd}$, reaccommodation by the observer can introduce up to $2 \%$ luminance contrast into an equiluminant display, but had this artifact been responsible for the perception of stereomotion, there would have been the stereomotion threshold elevation that is characteristic of luminance stimuli.

The lack of stereomovement threshold elevation distinguishes chromatic from achromatic stereomovement, and suggests that stereoscopic changes are processed separately for chrominance and luminance channels. This amplifies the previously established chrominance/luminance distinction in temporal characteristics (Kelly, 1975; Regan \& Tyler, 1971a, 1971b; van der Horst, 1969), and it supports the idea of separate pathways for the two types of stimulus. The pathways differ both at the level of chromatic combination (presumably in the retina, as discussed above) and at the level of binocular combination, which must occur in the cortex.

In the luminance pathway, there is lateral inhibition between cones of the same type, but not across types, as was suggested by the "perceptive field" results of Ransom-Hogg and Spillman (1980) and earlier studies (Bender, 1973; McKee \& Westheimer, 1970). But there are also clear functional reasons to exclude intertype inhibition between cones. It would reduce sensitivity for the detection of even local stimuli, and it would also introduce chromatic opponency into the luminance signals. Instead, it is reasonable to reserve such opponency with low sensitivity for specifically chromatic connections (upper part of Figure 6). Here we have a direct red/green pathway with opponent inhibition limited specifically to intertype connections, replacing the lateral intratype connections. For completeness, a possible connectivity for blue/yellow opponency is shown, with inhibition from a blue cone onto a summing junction between red and green cones. Note that this must be a separate junction; otherwise, there would be no distinction between each type of chromatic inhibition, and the chrominance channel would be onedimensional rather than two-dimensional.

This scheme has the virtue of producing lateral and chrominance interactions from alternative connectivity of the same mechanism, such that the two are mutually exclusive, thus explaining why chromatic sensitivity shows no evidence for inhibition and vice versa. All the interactions depicted require only one or two postsynaptic connections and could, in fact, take place inside the cone pedicle (assuming input from longer range horizontal cells).

\section{REFERENCES}

ANSTIs, S. M. (1970). Phi movement as a subtraction process. Vision Research, 10, 1411-1430.

Anstis, S. M., CavanaGh, P. (1983). A minimum motion technique for judging equiluminance. In J. D. Mollon \& L. T. Sharpe (Eds.),
Color vision: Physiology and psychophysics (pp. 155-166). London: Academic Press.

Arditi, A. R., Anderson, P., \& Movshon, J. A. (1981). Monocular and binocular detection of moving sinusoidal gratings. Vision Research, 21, 329-336.

BENDER, B. G. (1973). Spatial interactions between the red- and greensensitive colour mechanisms of the human visual system. Vision Research, 13, 2205-2218.

Cavanagh, P., Tyler, C. W., Favreau, O. E. (1984). Perceived velocity of moving chromatic gratings. Journal of the Optical Society of America, A1, 893-899.

Comerford, J. P. (1974). Stereopsis with chromatic contours. Vision Research, 14, 975-982.

DeYoe, E. A., \& VAN Essen, D .C. (1988). Concurrent processing streams in monkey visual cortex. Trends in Neuroscience, 11, 219-226.

Kaplan, E., \& Shapley, R. M. (1986). The primate retina contains two types of ganglion cells, with high and low contrast sensitivity. Proceedings of the National Academy of Sciences of the United States of America, 83, 2755-2757.

Kelly, D. H. (1971). Theory of flicker and transient responses: I. Uniform fields. Journal of the Optical Society of America, 61, 537-546.

KELLY, D. H. (1975). Luminous and chromatic flickering gratings have opposite effects. Science, 188, 371-372.

KELLY, D. H. (1979). Motion and vision: II. Stabilized spatiotemporal threshold surface. Journal of the Optical Society of America, 69, 1340-1349.

LAWTON, T. B., TYLER, C. W. (1986a). The role of lateral inhibition in determining the shape of the contrast transducer function. Journal of the Optical Society of America A, 3, 98.

LAWTON, T. B., \& TYLER, C. W. (1986b). The role of X and simple cells in the contrast transducer function. Investigative Ophthalmology and Visual Science Supplement, 27, 340.

Livingstone, M. S., \& Hubel, D. H. (1987). Psychophysical evidence for separate channels for the perception of form, color, movement and depth. Journal of Neuroscience, 7, 3416-3468.

LU, C., \& Fender, D. H. (1972). The interaction of color and luminance in stereoscopic vision. Investigative Ophthalmology, 11, $482-490$.

McKeE, S. P., \& Westheimer, G. (1970). Specificity of cone mechanisms in lateral interaction. Journal of Physiology, 206, 117-128.

Norcia, A. M., Wesemann, W., MANny, R. (1988). Sweep VEP vernier acuity and its cortical magnification. Investigative Ophthalmology \& Visual Science Supplement, 29, 370.

Ramachandran, V. S., \& GREgory, R. L. (1978). Does colour provide an input into human motion perception? Nature, 275, 55-57.

Ransom-Hogg, A., Spillman, L. (1980). Perceptive field size in fovea and periphery of the light and dark adapted retina. Vision Research, 20, 221-228.

REgAN, D. M., \& BEVERLEY, K. I. (1973). Some dynamic features of depth perception. Vision Research, 13, 2369-2379.

Regan, D. M., \& TYleR, C. W. (1971a). Some features of dynamic color vision. Vision Research, 11, 1307-1324.

Regan, D. M., \& Tyler, C. W. (1971b). Temporal summation and its limit for wavelength changes: An analog of Bloch's law for color vision. Journal of the Optical Society of America, 61, 1414-1421.

Robson, J. G. (1966). Spatial and temporal contrast sensitivity functions of the visual system. Journal of the Optical Society of America, 56, 1141-1142.

Schiller, P. H., Charles, E. R., \& Logothetis, N. K. (1989). The functions of color-opponent (C-O) and broad-band (B-B) channels in perception at isoluminance. Investigative Ophthalmology \& Visual Science Supplement, 30, 323.

Sclar, G., Maunsell, J., \& Lennie, P. (1990). Coding of the image contrast in central visual pathways of the macaque monkey. Vision Research, 30, 1-10.

Stromeyer, C. F., III, Klein, S. A. (1974). Spatial frequency channels in human vision as asymmetric (edge) mechanisms. Vision Research, 14, 1409-1420. 
TYLER, C. W. (1971). Stereoscopic depth movement: Two eyes less sensitive than one. Science, 174, 958-961.

TYLER, C. W. (1974). Induced stereomovement. Vision Research, 24, 609-613.

TYLER, C. W. (1975). Characteristics of stereomovement suppression Perception \& Psychophysics, 17, 225-230.

TYLER, C. W. (1978). Hyper-resolution in human perception of movement in visual displays. Proceedings of the Society of Photo-Optical Instrumentation Engineers, 19, 121-125.
Tyler, C. W., \& Foley, J. M. (1975). Stereomovement suppression for transient disparity changes. Perception, 3, 287-296.

VAN DER Horst, J. G. C. (1969). Chromatic flicker. Joumal of the Optical Society of America, 59, 1213-1217.

ZEKI, S. M. (1978). Uniformity and diversity of structure and function in rhesus monkey striate cortex. Journal of Physiology, 277, 273-290.

(Manuscript received March 29, 1990;

revision accepted for publication August 3, 1990.) 